\title{
Electrostatic Control of lons and Molecules in Nanofluidic Transistors
}

\author{
Rohit Karnik,,,$\| l$ Rong Fan,,,$\| l$ Min Yue, ${ }^{\dagger}$ Deyu Li, ${ }^{\dagger}$ Peidong Yang, ${ }^{, \neq, \S}$ and \\ Arun Majumdar ${ }^{\star, t}, \S$
}

\begin{abstract}
Department of Mechanical Engineering and Department of Chemistry, University of California, Berkeley, California 94720, and Materials Sciences Division, Lawrence Berkeley National Laboratory, Berkeley, California 94720
\end{abstract}

Received March 14, 2005

\begin{abstract}
We report a nanofluidic transistor based on a metal-oxide-solution (MOSol) system that is similar to a metal-oxide-semiconductor field-effect transistor (MOSFET). Using a combination of fluorescence and electrical measurements, we demonstrate that gate voltage modulates the concentration of ions and molecules in the channel and controls the ionic conductance. Our results illustrate the efficacy of field-effect control in nanofluidics, which could have broad implications on integrated nanofluidic circuits for manipulation of ions and biomolecules in sub-femtoliter volumes.
\end{abstract}

Nanofluidic devices, such as protein ion channels and inorganic pores and channels, have been used for highly sensitive biomolecular sensing down to the single-molecule level, ${ }^{1-4}$ separation of DNA, ${ }^{5,6}$ and as microfluidic interconnects. ${ }^{7}$ Charge-related effects such as concentration enhancement $^{8}$ and effect of surface charge on ionic conductance ${ }^{9}$ have also been reported. All these single nanofluidic channel/ pore devices transport ionic or molecular species passively through the nanochannel, simply like electron transport through a two-terminal device such as a resistor. Analogous to metal-oxide-semiconductor field-effect transistors ${ }^{10}$ (MOSFETs), introducing field effect modulation of ionic or molecular species in micro/nanofludic systems would promote them to a higher level of controllability and even logic operation. This level of control is highly desired and could advance the development of large-scale nanofluidic circuits.

In an electrolyte solution, counterions accumulate near a charged surface and co-ions are electrostatically repelled. ${ }^{11}$ Due to this counterion shield, the electric potential decays to its bulk value over a characteristic length known as the Debye length. The Debye length, $l_{\mathrm{D}}$, decreases as the ion concentration, $n$, increases, $l_{\mathrm{D}} \propto n^{-1 / 2}$, and is typically $1-100$ $\mathrm{nm}$ for aqueous solutions. In microchannels, the Debye length is usually much smaller than the channel dimensions, and the bulk of the solution is shielded from the surface charge (Figure 1a). Therefore, although interfacial effects such as

* Corresponding authors: p_yang@uclink.berkeley.edu; majumdar@ me.berkeley.edu.

Department of Mechanical Engineering, UC, Berkeley.

Department of Chemistry, UC, Berkeley.

$\S$ Lawrence Berkeley National Laboratory.

" These authors contributed equally to this paper. a

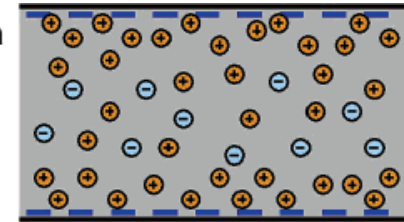

Microchannel

b

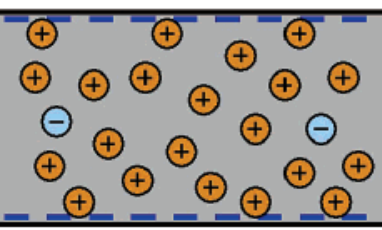

Nanochannel
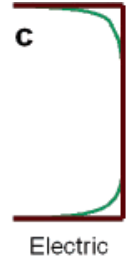

Potential

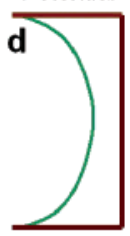

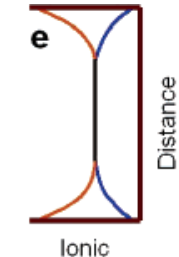

ncentration

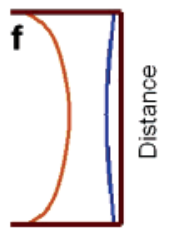

Figure 1. Surface charge effects in microchannels and nanochannels. (a) In a microchannel, the Debye length is typically much smaller than the channel dimensions and most of the solution in the channel is neutral. (b) In a nanochannel, the solution is charged when the Debye length is larger than the channel dimensions. (c) The electric potential in the microchannel decays rapidly to its bulk value in a distance of the order of the Debye length. (d) The electric potential even at the center of the nanochannel is influenced by the surface charge and is not equal to the bulk potential. (e) The concentration of cations (orange) and anions (blue) in the microchannel is equal to the bulk concentration. (f) In a nanochannel, the counterion concentration (orange) is much higher than the coion concentration (blue).

electroosmotic flow can be controlled using field-effect and surface modification in microchannels, ${ }^{12}$ direct electrostatic manipulation of ions across the microchannel is not possible. However, in nanochannels with at least one dimension 
comparable to or smaller than the Debye length, electrostatic fields can penetrate throughout the channel (Figure 1b), enabling direct ionic/molecular manipulation using surface charge or field-effect in such nanochannels. Martin et al. employed nanotubule membranes, which are essentially an array of nanochannels, for ion separation,,$^{13}$ detection, ${ }^{14}$ and sizing. ${ }^{15}$ They have demonstrated ion transport selectivity depending on the charge effect in metal nanotubule membranes, ${ }^{13,16}$ which suggests the feasibility of developing an ionic field effect transistor in nanofluidic systems similar to MOSFETs. Modeling of metal-oxide solution systems in silica nanotubes also shows a change in conductance on the application of a gate voltage. ${ }^{17}$ In addition, on-chip integration of single nanochannels is advantageous for constructing networks that combine in-situ optical probing of analytes. An early work over a decade ago investigated the change of electrical conductance of glycerol-filled channels, ${ }^{18}$ but their device had an extremely long relaxation time and did not allow for optical investigation. Here we demonstrate rapid field-effect control of ionic concentrations and conductance in nanofluidic transistors (nanochannels with gate electrodes) in sub-femtoliter volumes and provide direct evidence using fluorescence techniques.

Figure 2 shows nanofluidic transistors illustrating two types of fluidic confinement used in this study: (i) twodimensional silicon dioxide nanochannels that are $30-40 \mathrm{~nm}$ in height and $1 \mu \mathrm{m}$ wide (Figure 2b); (ii) one-dimensional silicon dioxide nanotubes that have internal diameters 10$100 \mathrm{~nm}$ (Figure 2c). The former devices are made completely by optical lithography, while the latter devices rely on separate synthesis of nanotubes ${ }^{19}$ and their subsequent integration with microfabricated channels and gate electrodes (see Supporting Information). $\mathrm{Ag} / \mathrm{AgCl}$ electrodes were used in the microfluidic channels/chambers on either side of the nanofluidic channels for applying electrical bias and generating ionic current.

For a two-dimensional nanochannel, the ionic current under source-to-drain electrical bias can be calculated as a superposition of conductive and convective contributions ${ }^{20}$

$$
I=\int_{-h}^{h} w e\left[\left(n_{+}+n_{-}\right) \mu E+\left(n_{+}-n_{-}\right) u\right] \mathrm{d} x
$$

where the integration is across the channel cross-section ( $x$ direction), $w$ is the width of the nanochannel, $2 h$ is the height and $\mu$ is the ionic mobility. The ionic concentrations $\left[n_{+}(x, z)\right.$, $\left.n_{-}(x, z)\right]$ and the potential $[\varphi(x, z)]$ distribution can be obtained by solving the coupled Poisson-Boltzmann equations, ${ }^{11,20}$ and the fluid velocity $u$ can be obtained by solving the Navier-Stokes equation. ${ }^{20}$ At low bulk ionic concentrations $(n)$, when $n \ll \sigma / e h$, where $\sigma$ is surface charge, counterions accumulate in the nanochannel to neutralize surface charge such that $n_{-} \approx \sigma e h$, and conductance is governed by surface charge. ${ }^{9}$ Figures $3 \mathrm{a}, \mathrm{b}$ show the measured ionic conductance of the nanochannel and nanotube devices along with theoretical predictions, which confirm surface-chargegoverned transport in our devices. The experiments tend to agree with conductance measurements reported recently, ${ }^{9}$ although with some deviations, presumably due to variation

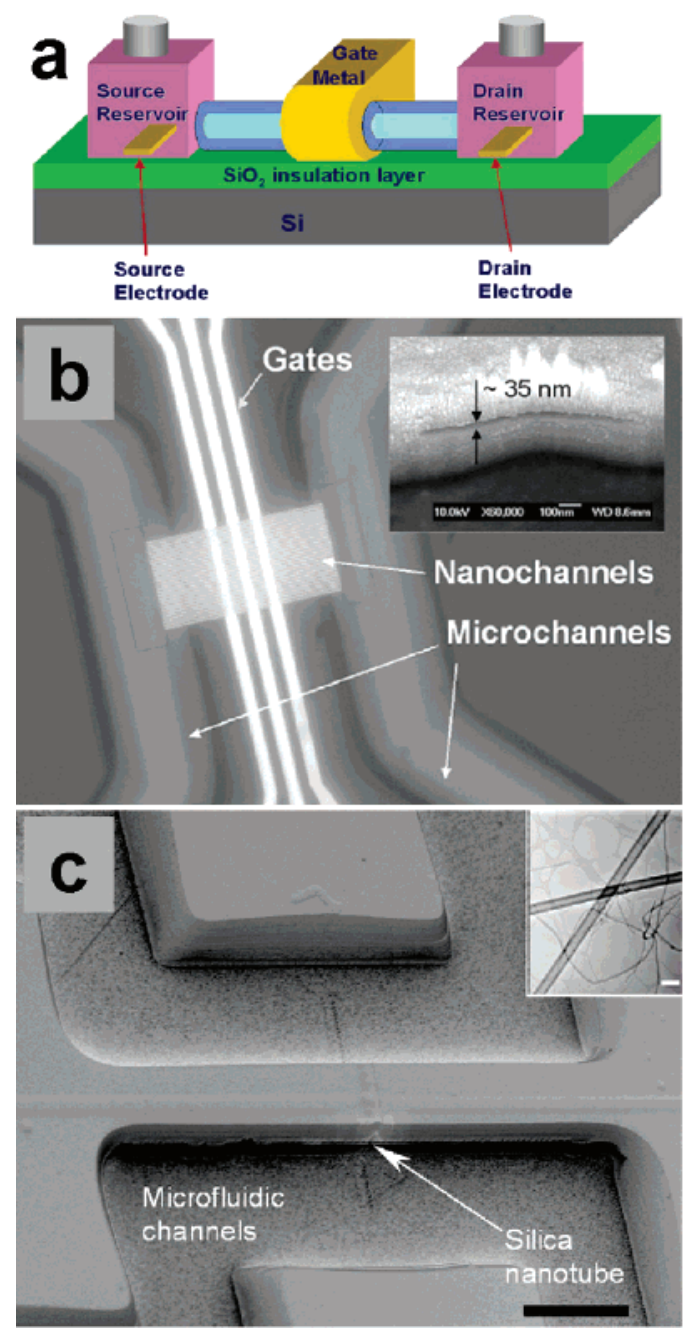

Figure 2. Nanofluidic transistor devices. (a) Schematic of a nanofluidic transistor. (b) Micrograph of the two-dimensional nanochannel transistor. Thirty nanochannels, $120 \mu \mathrm{m}$ long, run left to right and are connected by two microchannels. Three gate electrodes run vertically across the nanochannels. Nanochannels are made by etching a $35 \mathrm{~nm}$ thick and patterned poly-silicon layer (inset). (c) Scanning electron micrograph (SEM) of a onedimensional single nanotube transistor before bonding with the PDMS cover. The device contains a metallic gate electrode covered by a silicon dioxide patterned film. The tube is connected on both ends to microfluidic channels. Scale bar $10 \mu \mathrm{m}$. Upper right inset is a transmission electron micrograph (TEM) of silica nanotubes made by partial oxidation of silicon nanowires followed by etching the remaining silicon core. Scale bar $200 \mathrm{~nm}$.

of surface charge density. ${ }^{21-23}$ For the two-dimensional nanochannel, the results indicate that the surface charge density ranges between 0.002 and $0.1 \mathrm{C} / \mathrm{m}^{2}$, while it is about 0.01 to $0.02 \mathrm{C} / \mathrm{m}^{2}$ for the one-dimensional nanotubes. The results are consistent with reported values of the surface charge density. ${ }^{9,24,25}$ It is worth noting that the data also suggest that the surface charge increases with increasing $\mathrm{KCl}$ concentration, which may be due to ionic adsorption ${ }^{22}$ or surface charge regulation ${ }^{25}$ due to proximity of the nanochannel surfaces. This effect seems to be absent in the conductance measurements reported for larger nanochannels and microchannels. ${ }^{9}$ 

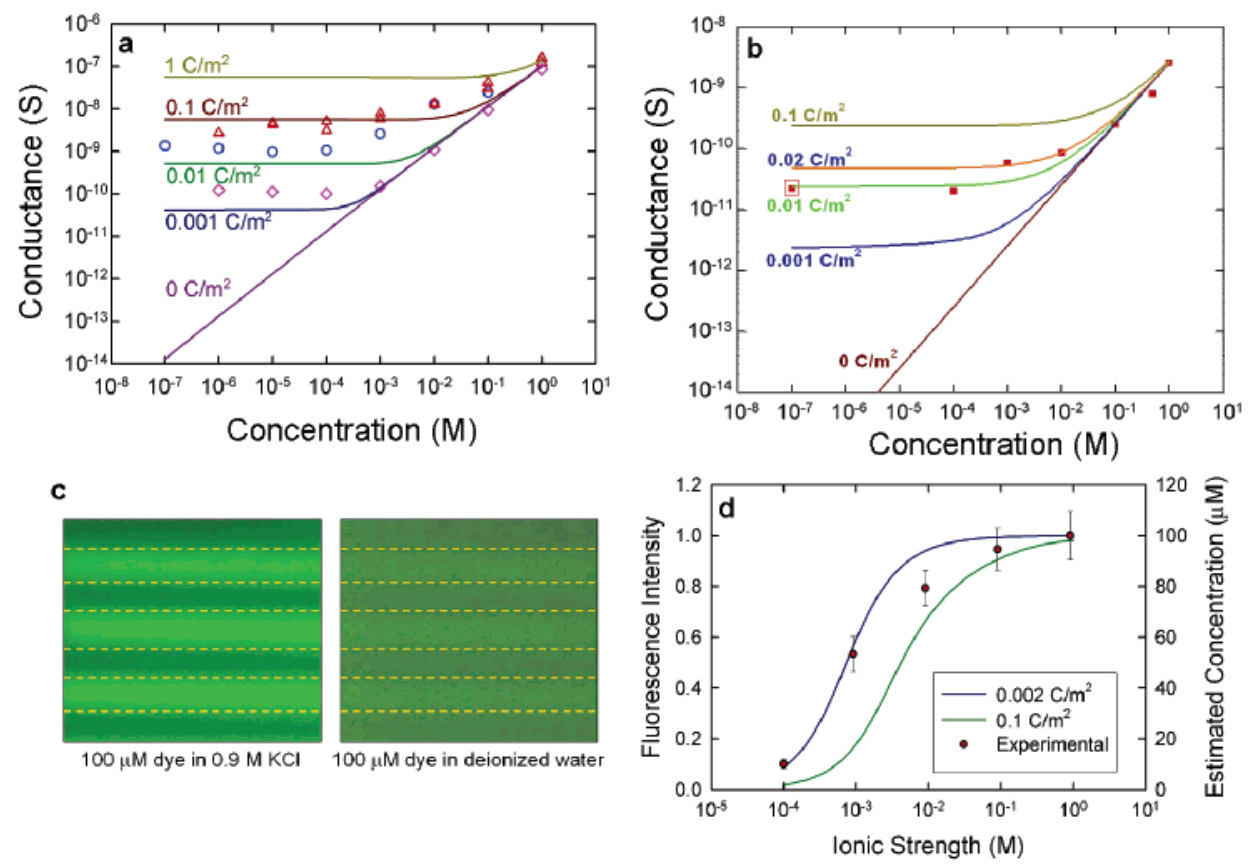

Figure 3. Electrostatic effects in nanochannels. The electrical conductance of the (a) nanochannel and the (b) nanotube devices deviates from that expected from bulk calculations $\left(0 \mathrm{C} / \mathrm{m}^{2}\right)$ by several orders of magnitude for low concentrations. Different symbols represent different devices in (a). Deionized water conductance in the nanochannels was also measured to give the data point at $10^{-7} \mathrm{M}$. This result is consistent with the theoretically calculated conductance with surface charge density as the parameter (solid lines indicated with respective surface charge densities). (c) Fluorescence images of a negatively charged dye (Alexa Fluor 488, monosodium salt) at $100 \mu \mathrm{M}$ concentration in deionized water and in $0.9 \mathrm{M} \mathrm{KCl}$. In deionized water, most of the dye molecules are excluded, while in $0.9 \mathrm{M} \mathrm{KCl}$, the dye molecules can enter the nanochannel because the surface charge is shielded. The dots outline three nanochannels, each $1 \mu \mathrm{m}$ wide. The images are enhanced and false-colored. Quantitative fluorescence intensity of the dye as a function of the ionic strength (d) shows a consistent decrease with decreasing ionic strength and is compared with theoretical predictions for a monovalent anion in a $35 \mathrm{~nm}$ channel, assuming a surface charge of $0.002 \mathrm{C} / \mathrm{m}^{2}$ and $0.1 \mathrm{C} / \mathrm{m}^{2}$. Assuming that the dye concentration in $0.9 \mathrm{M} \mathrm{KCl}$ represents the bulk value, the average dye concentration can be estimated for lower ionic strengths (right axis). The units of intensity are arbitrary. Intensity was measured over three $10 \mu \mathrm{m} \times 20$ $\mu \mathrm{m}$ areas with $\sim 10$ channels below the gates. Error bars are $1 \sigma$.

To further probe the electric potential in the nanochannels, a negatively charged dye (Alexa Fluor 488 cadaverine, monosodium salt) was introduced into the nanochannels. The bulk dye concentration was kept constant at $100 \mu \mathrm{M}$ while the $\mathrm{KCl}$ concentration was varied (Figure $3 \mathrm{c}, \mathrm{d}$ ). The chosen dye is insensitive to $\mathrm{pH}^{26}$ and, hence, the observed fluorescence intensity may be assumed to represent the actual amount of dye in the nanochannels. Strikingly, for low salt concentrations, the fluorescence intensity is one-tenth of that at high salt concentrations, in accordance with the theoretically predicted amounts of dye in the nanochannel (see Supporting Information). As the ionic strength decreases from 0.9 to $100 \mu \mathrm{M}$, the Debye length increases to about $30 \mathrm{~nm}$ and the effect of surface charge extends throughout the nanochannel. These results suggest that the magnitude of electric potential in the nanochannels is several times larger than $k T / e(26 \mathrm{mV})$ for low bulk concentrations. It implies that the concentration of cations is much larger than that of anions in the nanochannels.

A comparison with typical unipolar electronic devices, e.g., metal-oxide-semiconductor (MOS) systems, ${ }^{10}$ leads to the concept that a gate voltage may be employed to modulate the ionic concentration in nanochannels. This effect is similar to the field-effect modulation of carrier density in MOS systems via capacitive coupling between the gate electrode and the semiconductor. For low salt concentrations, external charges (including surface charge), such as those generated by an applied gate voltage, can affect the electric potential throughout the nanochannel when the Debye length is comparable to the channel size (Figure 4a). To examine the gating effect without any source-to-drain voltage bias, the negatively charged dye (Alexa Fluor 488) was introduced into the channels at a concentration of $100 \mu \mathrm{M}$ and the fluorescence intensity below the gate electrode was measured as a function of the gate voltage (Figure $4 b$ ). When a negative gate voltage was applied, the dye below the gate was repelled. On the other hand, the dye concentration was enhanced by a factor of 2 when a positive gate voltage of $50 \mathrm{~V}$ was applied. Since the oxide capacitance is small, assuming that the entire voltage drop occurs across the oxide, it results in a change in surface charge of roughly $3 \mathrm{mC} / \mathrm{m}^{2}$ for a gate voltage of $50 \mathrm{~V}$. The dye concentration estimated from the fluorescence intensity (Figure $4 \mathrm{~b}$ ) may be compared with the theoretically calculated dye concentrations of 75 , 30,18 , and $9 \mu \mathrm{M}$ at surface charge densities of $0.1,0.5,1$, and $2 \mathrm{mC} / \mathrm{m}^{2}$ respectively, suggesting that the observed change in intensity is in the expected order of magnitude. Moreover, theory predicts a highly nonlinear effect of surface charge density on the co-ion concentration, which is evident in the observed variation of intensity with gating voltage. For better control over ionic concentrations, a low surface charge, which determines the inherent ionic concentration 

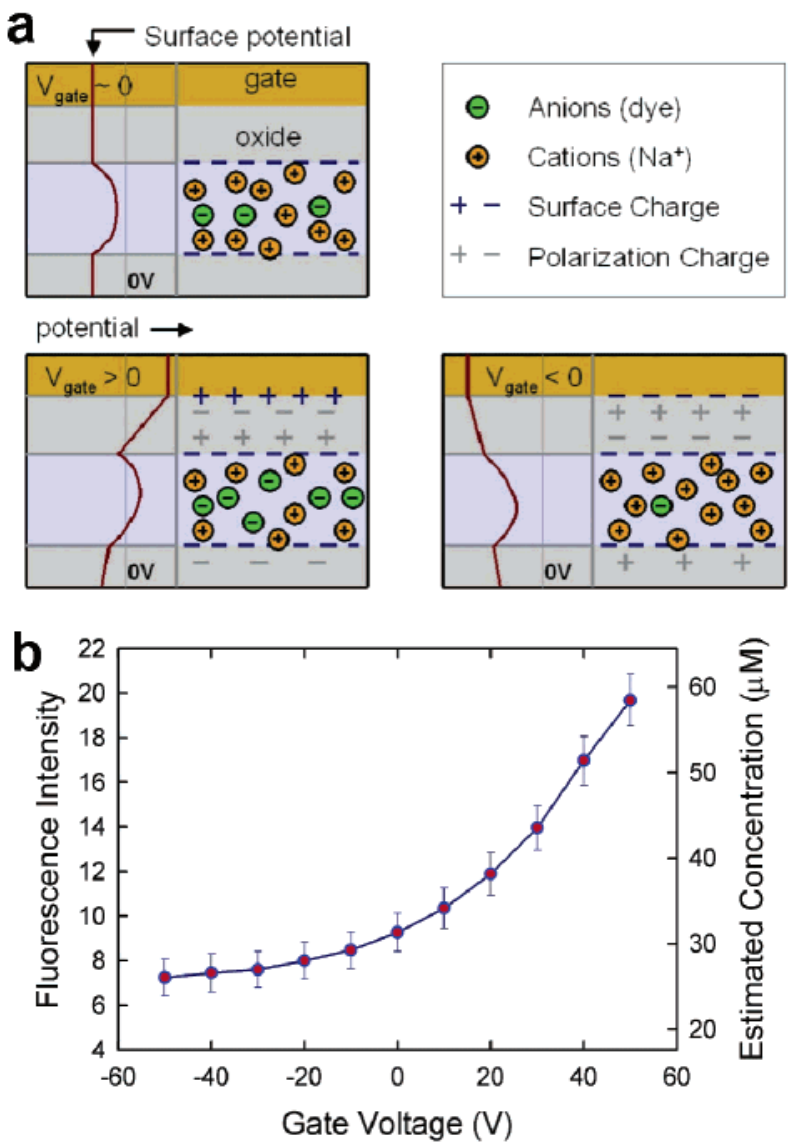

Figure 4. Field-effect modulation of ionic concentration in nanochannels. (a) Schematic illustration showing how the application of a gate voltage can control the ionic concentration. It is analogous to the carrier density modulation with the application of a gate voltage in a metal-oxide-semiconductor (MOS) structure. (b) The fluorescence intensity of a negatively charged dye (Alexa Fluor 488) can be controlled by the application of a gating voltage, thus demonstrating field-effect control in nanochannels. The average concentration of the dye in the nanochannel can be estimated assuming that the intensity at $0.9 \mathrm{M} \mathrm{KCl}$ represents the bulk dye concentration (right axis). Fluorescence intensity was measured over four $6 \mu \mathrm{m} \times 10 \mu \mathrm{m}$ areas below the gate electrode. Error bars are $1 \sigma$.

in the nanochannels, is desirable. It is worth noting that it is difficult to modulate carrier density in a MOS structure made of a degenerately doped semiconductor. Similarly, an inherent surface charge in nanochannels behaves like the high doping level in a semiconductor, making gating control of ionic concentration much more difficult.

To examine field-effect control of ionic conductance, the fluorescence intensity distribution of the negatively charged dye (Alexa Fluor 488) was imaged while a gate voltage was applied simultaneously with a source-to-drain voltage bias in the 2-dimensional nanochannel transistors. Controllable concentration gradients were observed below the gate electrodes; the direction of the concentration gradients could be switched by changing the gate voltage polarity (Figure 5a). This effect is analogous to the drain-induced barrier lowering (DIBL) ${ }^{10}$ in a MOSFET, which creates an asymmetric conduction channel and electric field distribution. The gate bias and the source-drain bias control the magnitude of a
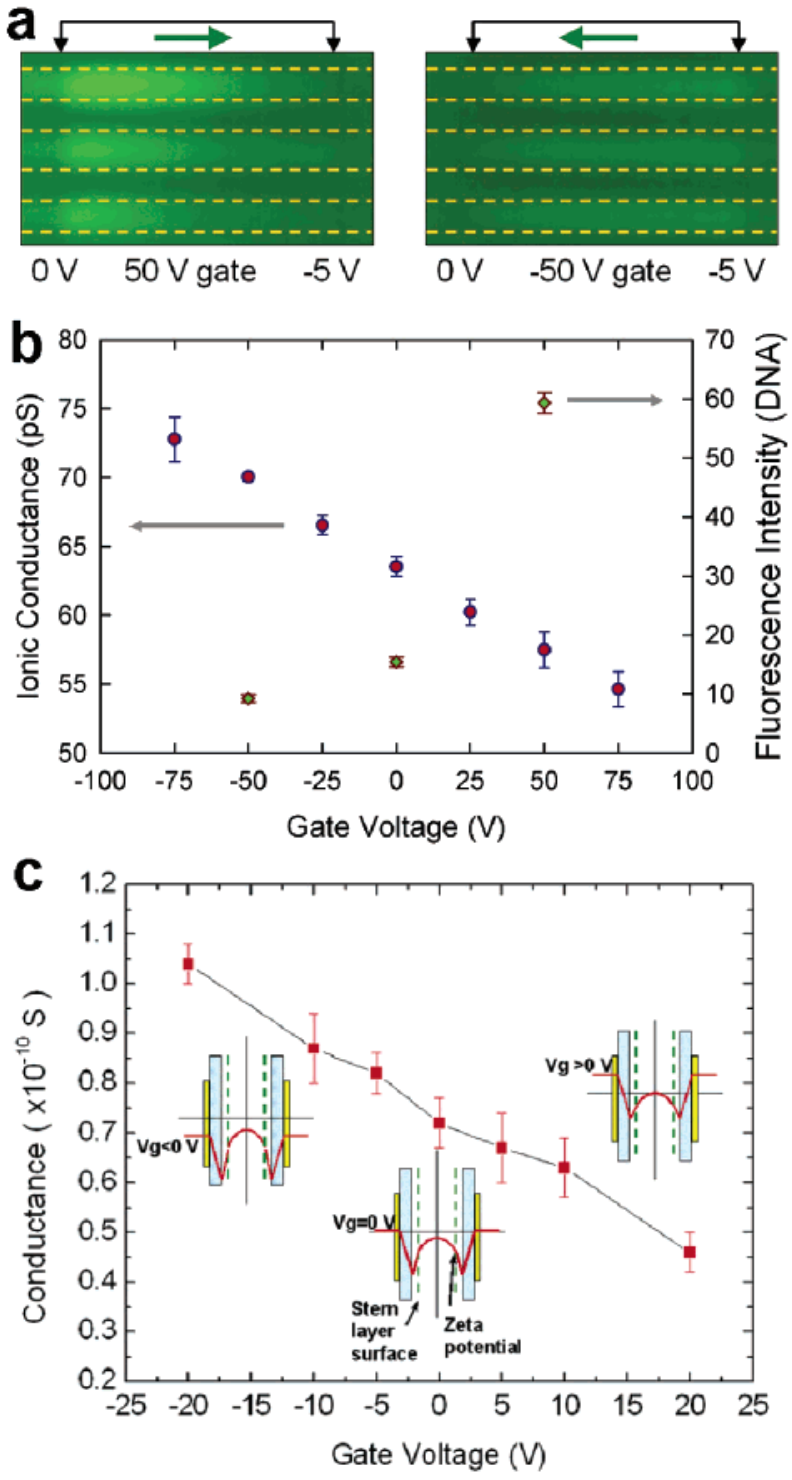

Figure 5. Field-effect control in nanofluidic transistors, which mimic the modulation of channel conductance in the metal-oxidesemiconductor field effect transistor (MOSFET). (a) Under a source-drain bias of $5 \mathrm{~V}$, gate voltage induces concentration gradients that switch directions with gate voltage polarity. Green arrows denote the concentration gradients; vertical arrows denote edges of the gate electrodes while the dots outline three nanochannels, each $1 \mu \mathrm{m}$ wide. Differential ionic conductance (slope of $I / V$ curves) of the nanochannels (b) and the nanotubes (c) can be modulated by a gate voltage. $100 \mu \mathrm{M} \mathrm{KCl}$ solution was used in this experiment. The insets schematically illustrate the electric potential from the gate electrode and across the nanotube when applying negative, zero, and positive gate voltages, which accordingly modulates ionic density and conductivity. The fluorescence intensity of fluorescein-labeled 30mer single-stranded DNA (ssDNA) molecules can be modulated by a factor of 6 by the application of a gate voltage (b), suggesting that flow control of charged biomolecules is feasible. The 30-base ssDNA concentration was $13.5 \mu \mathrm{M}$ in $1 \mathrm{mM} \mathrm{KCl}$. For the DNA experiment, fluorescence intensity was measured over four $6 \mu \mathrm{m} \times 10 \mu \mathrm{m}$ areas below the gate electrode. Units of intensity are arbitrary. Images are enhanced and false-colored. Error bars are $1 \sigma$.

the concentration enhancement: A gate bias of $50 \mathrm{~V}$ and a source-drain bias of $5 \mathrm{~V}$ led to a 10 -fold concentration enhancement. This phenomenon, which is electrokinetic in 
origin, reflects a balance between drift due to electrostatic and electroosmotic effects and diffusion due to concentration gradients. Similar phenomena have been theoretically described for current-carrying ion-selective membranes. ${ }^{27-29}$ The crucial difference here is that the ionic concentrations are both spatially and temporally controllable by electrostatic fields due to the microfabricated gate electrodes. This phenomenon could potentially be harnessed for applications such as isoelectric focusing of proteins ${ }^{30}$ and analyte stacking, ${ }^{31}$ while retaining the design flexibility of microfabrication and the controllability of gating voltage. As the gate voltage was changed from $-75 \mathrm{~V}$ to $75 \mathrm{~V}$, the ionic conductance of the nanochannels monotonically decreased (Figure 5b). Due to the inherent negative surface charge in the nanochannel, most of the current is carried by the cations. Hence, a negative gate voltage increases the cation concentration, thus increasing the conductance, whereas a positive gate voltage depletes the cations, resulting in a decrease in conductance.

Unlike the MOSFET, where the only function is to control electrical conductance, the nanofluidic transistor could be used to tune the ionic environment as well as to control the transport and concentrations of ions or particular charged biomolecular species. Since biomolecules are typically multivalent, gating control may be expected to be more effective for controlling biomolecules than for monovalent ions. We observed that the fluorescence intensity of 30-base fluorescently labeled single-stranded DNA (ssDNA) in a 1 mM KCL solution could be controlled by a factor of 6 in the nanochannels by the application of a gating voltage (Figure 5b). In deionized water, no fluorescence was observed, indicating that the DNA molecules were excluded due to their negative charge. However, in $1 \mathrm{mM} \mathrm{KCl}$, the surface charge was partially shielded, enabling gating control. Though the fluorescence intensity of fluorescein-labeled DNA is pH dependent, ${ }^{32}$ the 6-fold change in intensity cannot be accounted for by the approximately 2 -fold change in intensity due to $\mathrm{pH}$ variations. This result demonstrates the feasibility of electrostatic biomolecular control in nanofluidics.

Single inorganic nanotube nanofluidic transistors based on silica nanotubes were characterized for field-effect modulation of ion transport. The microchannels were bridged by a silica nanotube (nanotube wall thickness $\sim 35 \mathrm{~nm}$ ) which was covered by metallic surround gate electrodes. The $\mathrm{KCl}$ solution with low concentration $(\leq 1 \mathrm{mM})$ was used, which had a Debye length larger than $10 \mathrm{~nm}$. Nanotube nanofluidic transistors have two metallic surround gate electrodes. As shown in Figure 5c, gate voltage shifts the potential diagram across oxide, Stern layer and changes the effective surface charge density and $\zeta$ potential on the inner wall surface. When the nanotube size is comparable to Debye length, the ionic conductance depends only on $\xi$ potential and effective surface charge, which enables gate control of ionic conductance. In the experiment, ionic conductance was calculated from the slope of current-voltage $(I / V)$ curves by linear regression. Figure $5 \mathrm{c}$ shows the conductance at various gate voltages. For a gate voltage varying from $-20 \mathrm{~V}$ to $+20 \mathrm{~V}$, the ionic conductance decreases monotonically from $105 \mathrm{pS}$ down to $45 \mathrm{pS}$, due to depletion of cations under applied electric field, which shows a p-type transistor behavior.

In conclusion, we have clearly demonstrated that it is possible to electrostatically control ion transport in both 2-dimensional nanochannel transistors and single 1-dimensional nanotube transistors. If high dielectric constant materials are employed to fabricate the nanochannel wall, an enhanced field effect due to stronger capacitive coupling is expected. Moreover, when multivalent species such as biomolecules are present, the gating control over these molecules will be enhanced by the Boltzmann factor $\exp ((z$ $-1) e \varphi(k T)$, where $z$ is the number of charges on the species. Modifying the channel surface to obtain a low surface charge will further enhance the gating effect. The ability to spatially and temporally tune the ionic and electrostatic environment makes nanofluidic transistors a unique tool for biological and chemical analyses in sub-femtoliter volumes. The single nanotube transistors have the ability to manipulate and sense extremely small amounts of charged species, or even single biomolecules. Similar to metal-oxide semiconductor field effect transistors (MOSFETs), the nanofluidic transistor has the potential to form the building block of integrated nanofluidic circuits for manipulating biomolecules with single-molecule precision and control.

Acknowledgment. This work was supported by the IMAT program, National Cancer Institute, and the Office of Basic Energy Sciences, Department of Energy. We also thank Richard Cote, Ram Datar, Hirofumi Daiguji, and Andrew Szeri for their collaboration. A.M. would like to thank the Miller Institute for a Professorship. P.Y. is an A. P. Sloan Fellow. We thank the Microfabrication Laboratory (UC Berkeley) and the National Center for Electron Microscopy for the use of their facilities.

Supporting Information Available: Theoretical Analysis and Materials and Methods. This material is available free of charge via the Internet at http://pubs.acs.org.

\section{References}

(1) Kasianowicz, J. J.; Brandin, E.; Branton, D.; Deamer, D. W. Proc. Natl. Acad. Sci. U.S.A. 1996, 93, 13770-13773.

(2) Li, J.; Stein, D.; McMullan, C.; Branton, D.; Aziz, M. J.; Golovchenko, J. Nature 2001, 412, 166-169.

(3) Saleh, O. A.; Sohn, L. L. Nano Lett. 2003, 3, 37-38.

(4) Chang, H.; Kosari, F.; Andreadakis, G.; Alam, M. A.; Vasmatzis, G.; Bashir, R. Nano. Lett. 2004, 4, 1551-1554.

(5) Han, J.; Craighead, H. G. Science 2000, 288, 1026-1029.

(6) Foquet, M.; Korlach, J.; Zipfel, W.; Webb, W. W.; Craighead, H. G. Anal. Chem. 2002, 74, 1415-1422.

(7) Kuo, T.-C.; Cannon, D. M., Jr.; Chen, Y.; Tulock, J. J.; Shannon, M. A.; Sweedler, J. V.; Bohn, P. W. Anal. Chem. 2003, 75, 18611867.

(8) Pu, Q. S.; Yun, J. S.; Temkin, H.; Liu S. R. Nano Lett. 2004, 4, 1099-1103.

(9) Stein, D.; Kruithof, M.; Dekker, C. Phys. Rev. Lett. 2004, 93, 035901$1-4$.

(10) Sze, S. M. Physics of Semiconductor Devices, 2nd ed.; John Wiley and Sons: New York, 2002.

(11) Israelachvili J. Intermolecular and surface forces, 2nd ed; Academic Press: London, 2003.

(12) Schasfoort, R. B. M.; Schlautmann, S.; Hendrikse, J.; van den Berg A. Science 1999, 286, 942-945. 
(13) Nishizawa, M.; Menon, V. P.; Martin C. R. Science 1995, 268, 700702.

(14) Kobayashi, Y.; Martin C. R. Anal. Chem. 1999, 71, 36653672 .

(15) Jirage, K. B.; Hulteen, J. C.; Martin C. R. Science 1997, 278, 655658.

(16) Kang, M.-S.; Martin, C. R. Langmuir 2001, 17, 2753-2759.

(17) Daiguji H.; Yang, P.; Majumdar, A. Nano Lett. 2004, 4, 137142.

(18) Gajar, S. A.; Geis, M. W. J. Electrochem. Soc. 1992, 139, 28332840.

(19) Fan, R.; Wu, Y.; Li, D.; Yue, M.; Majumdar, A.; Yang P. J. Am. Chem. Soc. 2003, 125, 5254-5255.

(20) Levine, S.; Marriott, J. R.; Robinson K. J. Chem. Soc.-Faraday Trans. 2 1975, 71, 1-11.

(21) Parks, G. A. Chem. Rev. 1965, 65, 177-198.

(22) Yates, D. E.; Levine, S.; Healy T. W. J. Chem. Soc.-Faraday Trans. 1 1974, 70, 1807-1818.
(23) Grieser, F.; Lamb, R. N.; Wiese, G. R.; Yates, D. E.; Cooper, R.; Healy, T. W. Radiat. Phys. Chem. 1984, 23, 43-48.

(24) Sonnefeld, J. J. Colloid Interface Sci. 1996, 183, 597-599.

(25) Behrens, S. H.; Grier, D. G. J. Chem. Phys. 2001, 115, 67166721.

(26) Alexa Fluor dyes: http://www.probes.com/handbook/sections/ 0103.html, 2004.

(27) Oren, Y.; Litan, A. J. Phy. Chem. 1974, 78, 1805-1811.

(28) Fíla, V.; Bouzek, K. J. Appl. Electrochem. 2003, 33, 675-684.

(29) Zabolotsky, V. I.; Manzanares, J. A.; Nikonenko, V. V.; Lebedev, K. A.; Lovtsov, E. G. Desalination 2002, 147, 387-392.

(30) Righetti P. G. Isoelectric focusing: theory, methodology and applications; Elsevier: Amsterdam, 1983.

(31) Jung, B.; Bharadwaj, R.; Santiago, J. G. Electrophoresis 2003, 24, 3476-3483

(32) Sjöback, R.; Nygren, J.; Kubista, M. Biopolymers 1998, 46, 445-453. NL050493B 